\title{
Prenatal phenotype of PNKP-related primary microcephaly associated with variants affecting both the FHA and phosphatase domain
}

\author{
Sonja Neuser (iD ${ }^{1,10^{凶}}$, Ilona Krey (iD ${ }^{1,10}$, Annemarie Schwan ${ }^{2}$, Rami Abou Jamra (iD ${ }^{1}$, Tobias Bartolomaeus (iD ${ }^{1}$, Jan Döring ${ }^{3}$, \\ Steffen Syrbe ${ }^{3}$, Margit Plassmann ${ }^{4}$, Stefan Rohde ${ }^{5}$, Christian Roth $^{6}$, Helga Rehder ${ }^{7,8}$, Maximilian Radtke ${ }^{1}$, Diana Le Duc ${ }^{1}$, \\ Susanna Schubert ${ }^{1}$, Luis Bermúdez-Guzmán (D) ${ }^{9}$, Alejandro Leal $\mathbb{D}^{9}$, Katharina Schoner ${ }^{8}$ and Bernt Popp (iD ${ }^{1 凶}$
}

(c) The Author(s) 2021

Biallelic PNKP variants cause heterogeneous disorders ranging from neurodevelopmental disorder with microcephaly/seizures to adult-onset Charcot-Marie-Tooth disease. To date, only postnatal descriptions exist. We present the first prenatal diagnosis of PNKP-related primary microcephaly. Pathological examination of a male fetus in the 18th gestational week revealed micrencephaly with extracerebral malformations and thus presumed syndromic microcephaly. A recessive disorder was suspected because of previous pregnancy termination for similar abnormalities. Prenatal trio-exome sequencing identified compound heterozygosity for the PNKP variants c.498G >A, p.[(=),0?] and c.302C>T, p.(Pro101Leu). Segregation confirmed both variants in the sister fetus. Through RNA analyses, we characterized exon 4 skipping affecting the PNKP forkhead-associated (FHA) and phosphatase domains (p.Leu67_Lys166del) as the predominant effect of the paternal c.498G $>$ A variant. We retrospectively investigated two unrelated individuals diagnosed with biallelic PNKP-variants to compare prenatal/postnatal phenotypes. Both carry the splice donor variant c.1029+2T>C in trans with a variant in the FHA domain (c.311T>C, p.(Leu104Pro); c.151G >C, p.(Val51Leu)). RNA-seq showed complex splicing for $c .1029+2 T>C$ and $c .151 \mathrm{G}>C$. Structural modeling revealed significant clustering of missense variants in the FHA domain with variants generating structural damage. Our clinical description extends the PNKP-continuum to the prenatal stage. Investigating possible PNKP-variant effects using RNA and structural modeling, we highlight the mutational complexity and exemplify a PNKP-variant characterization framework.

European Journal of Human Genetics (2022) 30:101-110; https://doi.org/10.1038/s41431-021-00982-y

\section{INTRODUCTION}

Microcephaly, or rather micrencephaly (abnormally small brain) in the narrow sense, is defined as an occipitofrontal circumference (OFC) below -2 SD of the mean for (gestational) age and sex and can occur in isolated form or in a syndromic context [1]. If detected prenatally, it is classified as primary microcephaly (PM) in contrast to secondary microcephaly developing after birth. Infections, traumata, ischemic events, exposure to teratogens, and genetic disorders are possible etiologies [1, 2]. As head growth depends on normal neuronal tissue proliferation, requiring continuous cell division, several genetic neurodevelopmental and neurodegenerative disorders are caused by variants affecting DNA repair genes, highlighting the importance of the pathways in neurogenesis [3].

The polynucleotide kinase 3'-phosphatase (PNKP) has a dual kinase/phosphatase function and is involved in the repair of both single- and double-strand DNA breaks [4, 5]. In 2010, biallelic pathogenic PNKP variants were reported to cause "microcephaly, seizures, and developmental delay" (MIM\# 613402) [6]. Over time, three additional neurological and neurodevelopmental diseases have been associated with PNKP variants. These range from "childhood-onset ataxia with oculomotor apraxia type 4" (MIM\# 616267) [7] to "developmental and epileptic encephalopathy, type 10" (MIM\# 613402) [8] and "adult-onset Charcot-Marie-Tooth disease, type 2B2" (MIM\# 605589) [9, 10].

Most pathogenic PNKP variants described so far are either truncating or located in the C-terminal kinase domain [11]. While genotype-phenotype correlations have been attempted and C-terminal variants have been implied to cause the milder adultonset diseases, no clear relation could yet be established. Instead, it has even been postulated that the pathogenic variants observed present with rather mild mutational effects, due to survivorship bias, and more damaging variants would result in non-viability [11].

Here, we describe the first prenatal identification of biallelic PNKP variants affecting the region between the $\mathrm{N}$-terminal forkhead-associated (FHA) domain and the phosphatase domain

\footnotetext{
${ }^{1}$ Institute of Human Genetics, University of Leipzig Medical Center, Leipzig, Germany. ${ }^{2}$ MVZ Dr. Eberhard \& Partner Dortmund, Dortmund, Germany. ${ }^{3}$ Department of Pediatrics, Hospital for Children and Adolescents, Heidelberg University Hospital, Heidelberg, Germany. ${ }^{4}$ Praxis für Pränatalmedizin, Dortmund, Germany. ${ }^{5}$ Department of Radiology and Neuroradiology, Klinikum Dortmund, Dortmund, Germany. ${ }^{6}$ Department for Pediatric Radiology, University of Leipzig Medical Center, Leipzig, Germany. ${ }^{7}$ Institute of Medical Genetics, Medical University Vienna, Vienna, Austria. ${ }^{8}$ Institute of Pathology, Department of Fetal Pathology, Philipps University Marburg, Marburg, Germany. ${ }^{9}$ Section of Genetics and Biotechnology, School of Biology, University de Costa Rica, San José, Costa Rica. ${ }^{10}$ These authors contributed equally: Sonja Neuser, Ilona Krey. ${ }^{凶}$ email: sonja.neuser@medizin.uni-leipzig.de; bernt.popp.md@gmail.com
} 
causative for severe early onset of PM. We provide detailed descriptions based on prenatal imaging and syndrome-oriented fetal autopsies of two affected sibling fetuses and compare the fetal phenotype with two individuals with PNKP-associated disorder and literature cases. In addition, we performed RNA analyses to characterize aberrant splicing of identified variants and used structural modeling to investigate missense variants.

\section{MATERIALS AND METHODS}

Genetic analyses and review of PNKP variants

P2 and both parents underwent trio-exome sequencing. In P3 and P4, clinical exome sequencing (CES) was performed. Segregation was confirmed through Sanger sequencing in all. Technical details and primer sequences are provided in Supplementary notes. All PNKP variants have been submitted to ClinVar (Supplementary File S3 [12] sheet "PNKP_variants").

A PubMed search using the term "PNKP" identified 38 distinct PNKP variants from 21 publications (searched on December 15, 2020, details in Supplementary File S3 [12] sheet "PNKP_variants"). Variants were standardized to the reference transcript NM_007254.3 (GRCh37/hg19) using Mutalyzer 2.0.32 and annotated as described previously [13] (see Supplementary File S3 [12] sheet "PNKP_variants"). PNKP variants were classified following ACMG guidelines [14].

\section{Clinical data collection}

We used a questionnaire for retrospective phenotypical analysis with clinical terms (standardized using HPO [15]) based on a review of clinical associations in PNKP-disorders [6-10]. The sheet was sent for evaluation to the pediatric neurologist or pathologist, respectively, and available clinical reports were added. Pre-/postnatal measurements were compared to published standards [16] or WHO child growth charts [17]. Comprehensive results are found in Supplementary File S2 [12] sheet "clinical" and Supplementary Fig. S1.

\section{Fetal autopsy and RNA extraction from fetal tissue}

Fetal pathological examination of P1 and P2 was performed as previously described [18] (details in Supplementary notes). Cryopreserved native skeletal muscle tissue of P2 was processed with QIAshredder (Qiagen, Hilden, Germany) and RNA was extracted according to the manufacturer's protocol (RNeasy Mini, Qiagen, Hilden, Germany).

\section{RNA analyses}

In family 1, we performed RT-PCR as described previously [19] using PAXgene RNA in the parents and fetal skeletal muscle RNA derived CDNA. In family 3, we performed RNA-seq from PAXgene RNA using the TruSeq RNA Library Prep Kit v2 and paired-end sequencing. Bioinformatic workup included an established pipeline from our institute. In brief, reads were demultiplexed, adapters trimmed, and overrepresented sequences removed before we aligned the reads to the hg38 reference. Alignments were visualized and inspected for aberrant splicing as described previously [20]. We applied iREAD [21] to quantify observed intron retention events. Please see details in the Supplementary notes.

\section{Analysis of missense variant spectrum}

Disease-associated missense analysis in the linear protein, clustering analysis in $3 \mathrm{D}$ and structural modeling of missense variants using the crystal structure 2BRF [22] was performed as described previously $[11,13,19]$ and is detailed in the Supplementary notes.

\section{RESULTS}

\section{Prenatal phenotype in two sibling fetuses}

In the third pregnancy of a healthy non-consanguineous couple, routine sonography at 13 weeks gestational age (GA) revealed microcephaly, abnormal skull shape, and microretrognathia in the male fetus (P2). Follow-up ultrasound (US) examinations at 15 and 16 weeks GA displayed progression of the anomalies. Amniocentesis for genetic testing (trio-ES) was performed at 16 weeks GA. Severe fetal anomalies and supposing genetic background caused the parents to decide for termination of pregnancy in week $18+3$.

A previous pregnancy had been terminated at 22 weeks GA after prenatal imaging had confirmed multiple anomalies in a female fetus
(P1). US at $19+1$ weeks GA had shown microcephaly, asymmetric skull shape, abnormal brain development, cerebellar hypoplasia, cataract of both eyes, and facial abnormalities. Prenatal MRI at 20 +6 weeks GA confirmed microcephaly, large supratentorial defects of brain parenchyma in occipital, parietal and frontal regions, severe cerebellar hypoplasia, and dilatation/fusion of both lateral ventricles (Fig. 1C). Corpus callosum and septum pellucidum were not determinable. Bulbi of the eyes differed in size and signal.

Both fetuses were examined after TOP. The female fetus P1 (TOP at 22 weeks GA) measured $24.0 \mathrm{~cm}$ crown to heel length (standard: 26.2 $\pm 3.6 \mathrm{~cm}$ [16]) having a weight of $301 \mathrm{~g}$ (standard: $353 \pm 125 \mathrm{~g} \mathrm{[16]).}$ OFC was $15.0 \mathrm{~cm}$ (-4.3 SD; 5th percentile at GA: $17.3 \mathrm{~cm}$ [23]). Autopsy showed severe cerebral parenchymatous defects, profound hypoplasia of posterior cranial fossa, and confluence of the side ventricles. The findings were initially interpreted as arhinencephaly/holoprosencephaly. Extracerebral anomalies were not assessable (autolysis). Placenta appeared hypotrophic with signs of insufficiency.

The male brother fetus P2 (TOP at $18+3$ weeks GA) was almost age-appropriate in terms of crown to heel length of $18.8 \mathrm{~cm}$ and weight $124.3 \mathrm{~g}$ (standard: $20 \mathrm{~cm}$ and $150 \mathrm{~g}$ ), but had extremely small OFC $(11.3 \mathrm{~cm},-5.85 \mathrm{SD}$; 5 th percentile at this GA: $14.1 \mathrm{~cm}$ [23]). Fetal autopsy confirmed severe micro-/brachycephaly, short receding forehead, narrow fontanelles, and associated facial dysmorphisms (hypertelorism, anteverted nares, long philtrum, small upper lip, small outer ears). Moreover, the fetus showed contractures according to early manifestation of arthrogryposis. Brain volume was reduced (about $40 \%$ of GA norm [16]) with slight enlargement of the ventricles. The frontal lobes were hypoplastic, occipital lobes were shortened and appeared winglike. Temporo-parietal lobulation and corpus callosum were missing. Cerebellum was hypoplastic with a diameter of $1.2 \mathrm{~cm}$ (5th percentile for GA: $1.6 \mathrm{~cm}$ [23]). All examined brain sections appeared histologically normal. The findings were interpreted as micrencephaly without neuronal migration disorder or structural malformations. Discrete anisophthalmia/anisocoria with partial lens luxation due to dysplasia of the iris and persistent hyaloid artery of the left eye was noticed. There were no signs for external causes and no further organ abnormalities. Fetal autopsy results of P2 were suspected as monogenic syndromic type of microcephaly.

\section{Genetic analyses}

Initial genetic investigations in P1, including conventional karyotyping, chromosomal microarray analysis, and a targeted holoprosencephaly sequencing panel were unremarkable. The recurrent pattern of PM in two successive pregnancies suggested a recessive syndromal type of PM. Thus, trio-ES was initiated after amniocentesis of P2. This analysis revealed the compound heterozygous $P N K P$ variants c.498G $>A$, p. $[(=)$, ?] and c.302C $>T$, p.(Pro101Leu). The paternal variant c.498G $>A$ is formally annotated as synonymous $(p .(=))$, but affects the last base of exon 4 and is predicted to disrupt the splice donor motif (p.(?)) with simple skipping of exon 4 resulting in an in-frame deletion between the FHA and the phosphatase domains. The maternal variant c.302C $>$ T causes a proline to leucine missense change at the amino acid (AA) position 101 in the FHA domain. Both were initially classified as variants of unknown significance (VUS) according to ACMG recommendations (criteria: PM2, PP3). Subsequent segregation analysis in an archived amniotic fluid sample confirmed these two variants in compound heterozygous state in the affected fetus P1. Despite co-segregation evidence supporting pathogenicity, the variants' classification remained VUS. Identified PNKP variants, variant effects, and classification are listed in Table 1. In P1 and P2, no other known pathogenic- or phenotypic-relevant variant was detected neither in PNKP nor in other disease-associated genes.

\section{Postnatal phenotype in two unrelated individuals}

The male index individual from family 2 (P3) was the first child of healthy non-consanguineous German parents with unremarkable family history. He was born at term after an uneventful pregnancy 

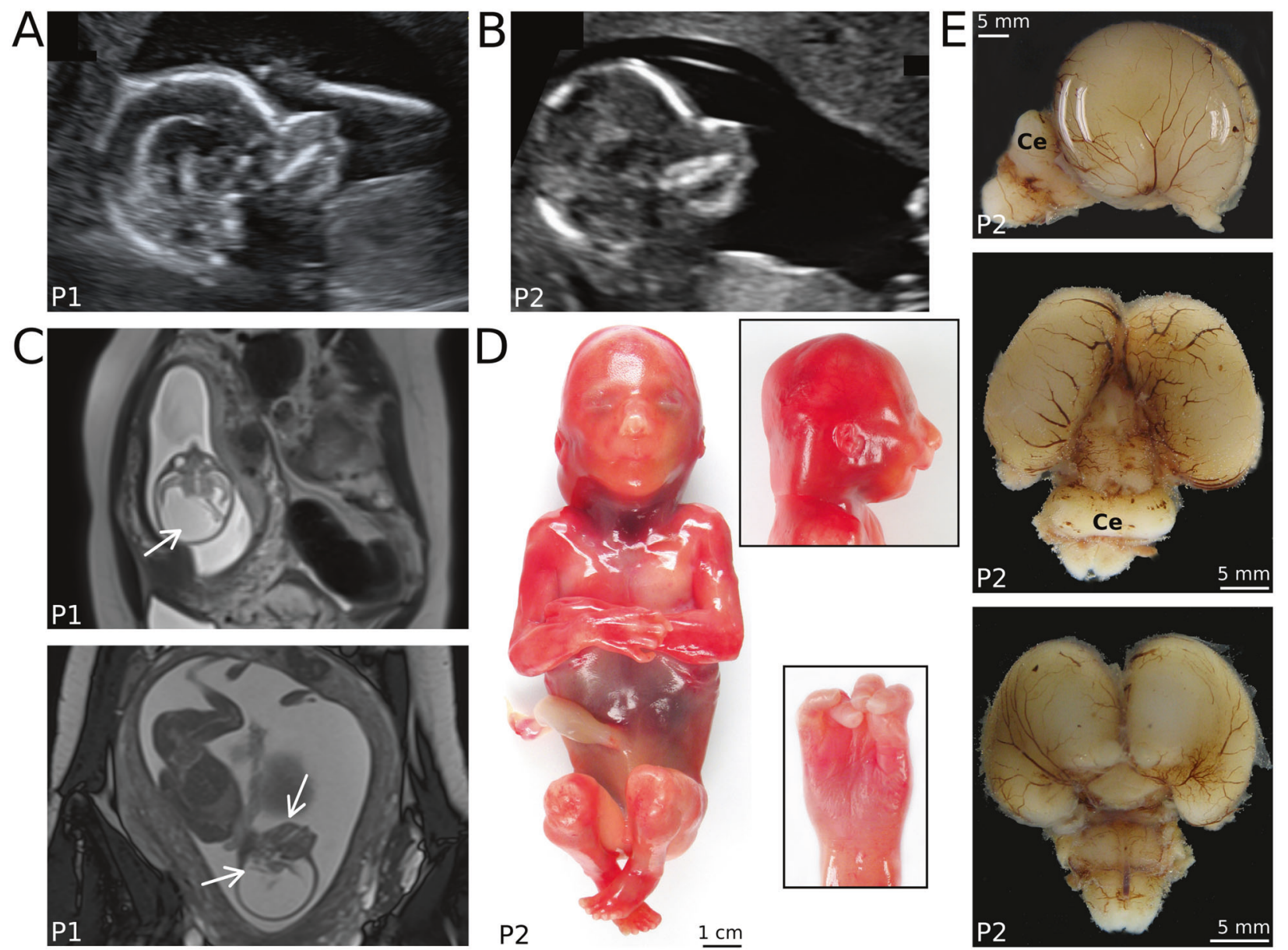

P2

$\underline{1 \mathrm{~cm}}$

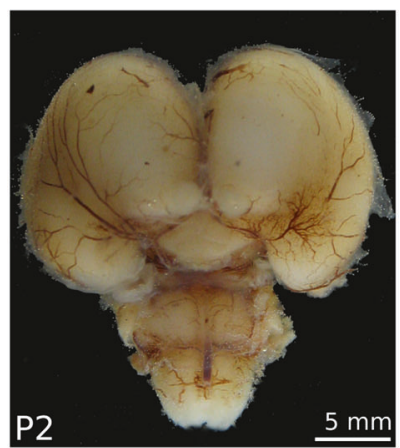

Fig. 1 Prenatal clinical and autopsy results. A Prenatal ultrasonography of female fetus P1 at GA $19+1$ weeks and B of male fetus P2 at GA 14 +3 weeks show comparable micro-/brachycephaly with accentuated flattened short forehead and retromicrognathia in sagittal plane. C T2 axial MRI image at GA $20+6$ weeks shows microcephaly and large supratentorial defects of the brain in occipital, parietal, and frontal regions with dilatation and fusion of both side ventricles. Lower panel: T2 TrueFISP sagittal image (GA 20+6 weeks) shows severe cerebellar hypoplasia as well as retrognathia. D Postmortem examination reveals the male fetus with appropriate development, arthrogryposis with camptodactyly (shown in detail), severe micro- and brachycephaly, short receding forehead, and retromicrognathia (shown in detail). E Brain in lateral, occipital, and frontal view presenting micrencephaly, especially affecting frontal lobes and moderate cerebellar hypoplasia (Ce).

with a weight of $2915 \mathrm{~g}(-0.92 \mathrm{SD})$, a length of $52.0 \mathrm{~cm}(+1.12 \mathrm{SD})$ and OFC of $31.5 \mathrm{~cm}(-2.33 \mathrm{SD})$. Prenatal sonography was reported as unremarkable at GA 13 and 22 weeks. Postpartal, he showed muscular hypotonia and trigonocephaly caused by frontal synostosis. At the age of 5 months, he developed therapy refractory focal motor seizures with impaired awareness, bilateral tonic seizures, and multiple status epilepticus. Seizures were partially responsive to oxcarbazepine and valproic acid. Individual P3 was last reviewed at the age of 2 years and 4 months. His height was $83 \mathrm{~cm}(-2.04$ SD) and weight was $8.4 \mathrm{~kg}(-4.49 \mathrm{SD})$. His OFC at the age of 2 years 6 months was $39 \mathrm{~cm}$ (-6.82 SD; Supplementary Fig. S1). Facial dysmorphism concerned typical stigmata of microcephaly. Neurological examination provided muscular hypotonia, ataxia, and oculomotor apraxia. He had moderate to severe global developmental delay with no speech and some limited passive understanding of words and signs but no active nonverbal communication. Developmental regression or behavioral abnormalities were not reported. Cranial MRI (cMRI) at the age of 5 months showed supra- and infratentorial white matter deficit, small corpus callosum, and a myelination delay. Brain imaging at the age of 2 years (Supplementary Fig. S2) showed extensive progressive microcephaly with simplified gyral pattern, an increasing white matter deficit, and the corpus callosum/cerebellum hypoplasia.

CES identified the two heterozygous PNKP variants c. $1029+2 T>C, p$. (?) and c.311T >C, p.(Leu104Pro). Segregation analysis in the parents confirmed compound heterozygosity. The maternal variant c.1029 $+2 \mathrm{~T}>\mathrm{C}$ affects the canonical splice donor in intron 11, likely causing aberrant mRNA splicing. Skipping would result in an in-frame deletion of the 93 base pairs (bp) of the adjacent exon 11 causing a deletion of $31 \mathrm{AAs}$ in the phosphatase domain. In fact, this variant was previously published, and RNA was analyzed using RT-PCR, which confirmed the predicted skipping effect [24]. The paternal base pair substitution c.311T $>C$ causes a leucine to proline missense change at the $A A$ position 104 in the FHA domain. The splice donor variant was initially classified as likely pathogenic while the missense variant was classified as VUS (criteria: PVS1, PM2 for c.1029+2T>C, p.(?); PM2, PM3 for c.311T>C, p.(Leu104Pro)).

The male index from family 3 (P4) was the first child of healthy non-consanguineous German parents with unremarkable family history. The pregnancy was complicated through a vanishing twin around GA 9 weeks, oligohydramnios, premature labor, and contractions at 17 and 24 weeks GA. Prenatal sonographic examination provided inconspicuous fetal development in gestational week 20. Individual P4 was born at term via caesarean section for arrest of labor with a weight of $3070 \mathrm{~g}(-0.58 \mathrm{SD})$, a length of 47 $\mathrm{cm}(-1.52 \mathrm{SD})$, and OFC of $32 \mathrm{~cm}(-1.94 \mathrm{SD})$. He presented with postnatal muscular hypotonia. At the age of 8 months, he showed motor development regression (loss of grabbing and turning). At age 12 months, he was able to speak first words and sit 
independently. At the last consultation at age 2 years and 9 months, his height was $86 \mathrm{~cm}(-2.32 \mathrm{SD})$, his weight was $11 \mathrm{~kg}(-2.36 \mathrm{SD})$, and his OFC was $42 \mathrm{~cm}(-5.14 \mathrm{SD})$, representing severe progressive microcephaly (Supplementary Fig. S1) and he had mild dysmorphic facial features (epicanthus, hypotelorism, and deep-set ears). Exploratory neurological examination showed muscular hypotonia and ataxia. He had mild global developmental delay. Brain imaging via CMRI and SPECT at the age of 2 years showed microcephaly without additional brain abnormalities (Supplementary Fig. S2).

CES revealed two heterozygous variants in $P N K P$, the heterozygous splice variant c. $1029+2 \mathrm{~T}>\mathrm{C}$, p.(?) and c.151G $>$ C, p. (Val51Leu). Sanger sequencing confirmed the presence of both variants in P4 and their heterozygosity in the parents. The canonical splice variant was inherited from the mother. The

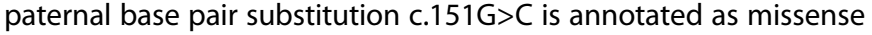
change p.(Val51Leu) in the FHA domain but also affects the last nucleotide of exon 2, potentially affecting mRNA splicing. The splice donor variant was again classified as likely pathogenic while the missense variant was classified as VUS (criteria: PVS1, PM2 for c.1029+2T>C, p.(?); PM2, PM3 for c.151G >C, p.(Val51Leu)). In P3 and P4, no other known pathogenic- or phenotypic-relevant variant was detected neither in PNKP nor in other diseaseassociated genes.

\section{Analysis of missense variants in the FHA domain}

Of 43 total unique variants reported here and in the literature (19 missense $\sim 44.2 \%$ and two in frame AA deletions $\sim 4.7 \%$ ), seven missense variants $(7 / 19, \sim 36.8 \%)$ are located in the FHA domain, four $(4 / 19, \sim 21.1 \%)$ in the phosphatase and eight $(8 / 19, \sim 42.1 \%)$ in the kinase domain. Mean values of the CADD scores regarding the FHA (22.2), phosphatase (23.5), and kinase (21.5) domains are significantly higher than for the linker domain ( $p<2 \mathrm{e}-16$, one-way ANOVA). These regions also contain all missense variants reported as (likely) pathogenic and disease-associated missense VUS. The linker stands out with a mean CADD score of 13.8 and the lack of diseaseassociated missense variants.

All three missense variants identified in P1-P4 are located in the FHA domain. Review of missense variants from the literature revealed four additional disease-associated variants in this domain (Fig. 2A). Beside the c.58C $>\mathrm{T}$, p.(Pro20Ser) variant, which we classified as likely benign due to homozygous occurrence in reference populations (gnomAD), all missense variants previously reported as disease-associated in the FHA domain were classified as VUS using automated ACMG interpretation. Manual curation led to an evaluation as likely pathogenic for c.302C $>T$, p. (Pro101Leu). Three missense variants outside the FHA domain were also evaluated as likely pathogenic (c.526C >T, p.(Leu176Phe); c.968C >T, p.(Thr323Met); c.976G>A，p.(Glu326Lys)). ACMG [14] classifications of all variants can be found in Supplementary File S3 [12], sheet "PNKP_variants."

The spatial distribution of AA residues affected by missense variants in the FHA domain (2BRF from RCSB Protein Data Bank) showed that most disease-associated missense variants affect conserved residues in beta sheets (Supplementary Fig. S3B). Analysis with mutation $3 D$ revealed clustering of the affected $A A$ positions 50, 51, 55, 66, 101, and 104 in the FHA domain with a significant $p$ value (bootstrapping) of 0.0112 (Fig. 2B, C).

According to Missense3D, the C.302C>T, p.(Pro101Leu) variant triggers a local steric clash alert (Fig. 2D). In addition, based on the DynaMut web server predictor, the effect of this variant is stabilizing and the $\Delta$ Vibrational Entropy Energy between wildtype and mutant structure is predicted to slightly decrease molecule flexibility (Fig. 2D). The c.311T>C, p.(Leu104Pro) substitution introduces a buried proline in the core of the protein domain, which tends to be particularly damaging with its restricted backbone conformation (Fig. 2E). In fact, according to DynaMut, the variant has the opposite effect of p.(Pro101Leu) and is predicted to be destabilizing with increase of the molecule 
A

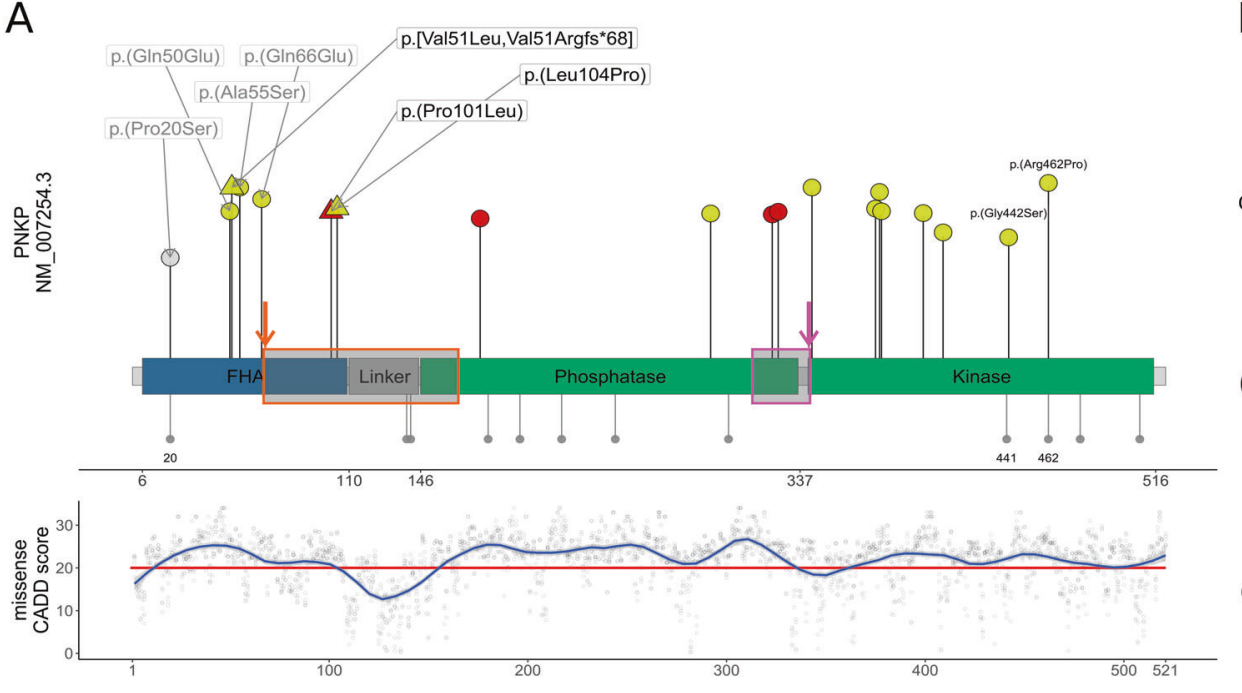

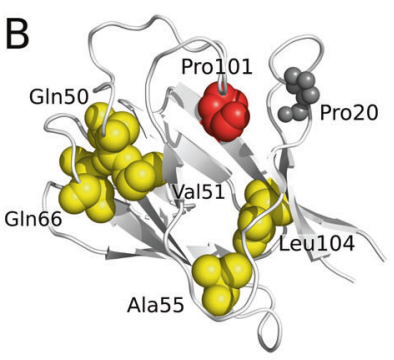

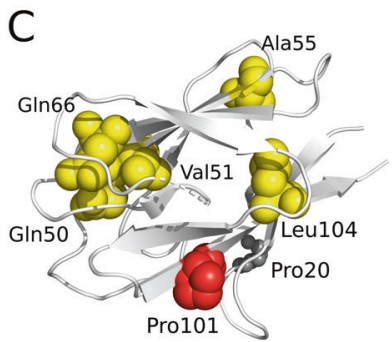

D
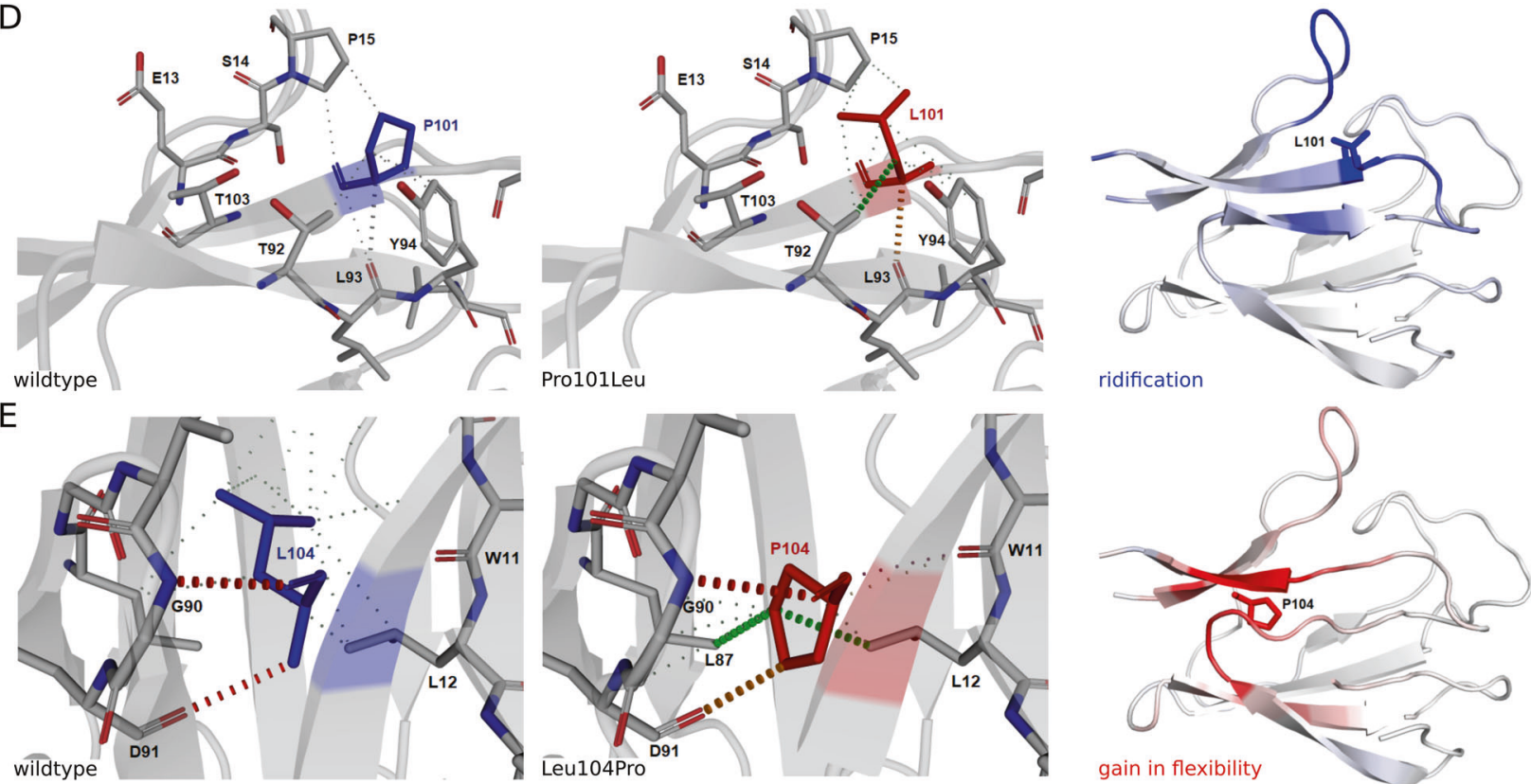

Fig. 2 Missense variants. A Schematic depiction of the PNKP protein with FHA in blue, Phosphatase and kinase domain in green and linker region in gray (based on Uniprot Q96T60 and [20]). Disease-associated missense variants are displayed toward the top, red shapes show (likely) pathogenic variants, yellow shapes show variants of unknown significance, and gray shapes show (likely) benign variants. The variants identified in the four individuals reported here are depicted as triangles (black labels), variants from the literature as dots (gray labels). The length of the segments corresponds to each variant's CADD score. Gray dots downwards show homozygous missense variants from gnomAD, the dot size represents the logarithm of the allele count. AA positions 20, 441, and 462 are labeled for orientation. In the panel below, a generalized additive model shows the CADD values for all possible missense variants (red horizontal line $=$ recommended cut-off [20]). Additional boxes show the affected regions of the in-frame deletions resulting from the synonymous variant $c .498 \mathrm{G}>\mathrm{A}$ in orange or the splicing variant c.1029+2T>C in pink. B, C Two representative views of the FHA domain crystal structure (based on 2BRF). AAs of missense variants are depicted in gray for benign variants (Pro20), in yellow for VUS (GIn50, Val51, Ala55, GIn66, and Leu104), and in red for pathogenic variants (Pro101). All missense variants classified as unknown significance or (likely) pathogenic affect conserved residues in beta sheets. D The substitution Pro101Leu alters the local interatomic interaction of the protein, generating important steric clashes at the same time it decreases molecule stability. The most right panel represents the $\Delta$ Vibrational Entropy Energy derived from the Pro101Leu variant, showing a local rigidification of the protein. E The Leu104Pro variant generates the opposite effect observed for Pro101Leu as it introduces a proline in the core of the FHA domain, destabilizing the protein domain. The most right panel shows the $\Delta$ Vibrational Entropy Energy with a local gain in flexibility.

flexibility (Fig. 2E). Comprehensive results for variants in the FHA domain are listed in Table 2.

\section{RNA analyses}

To determine the effect of the paternally inherited c.498G $>A$ variant in $\mathrm{P} 1$ and $\mathrm{P} 2$, we performed RT-PCR and Sanger sequencing on CDNA from RNA of individual P2 and both parents (F1-m, F1-f). In addition to the wildtype product, we identified a second, smaller PCR product in the sample of the father and P2. Sanger sequencing of this smaller product revealed skipping of the 300 base pair long exon 4 (r.199_498del) leading to an in-frame deletion of the 100 AAs of exon 4 on protein level (p.Leu67_Lys166del) (Fig. 3A).

To elucidate the effect of the paternal base pair substitution c.151G >C, affecting the last base of exon 2, and proof the recently 

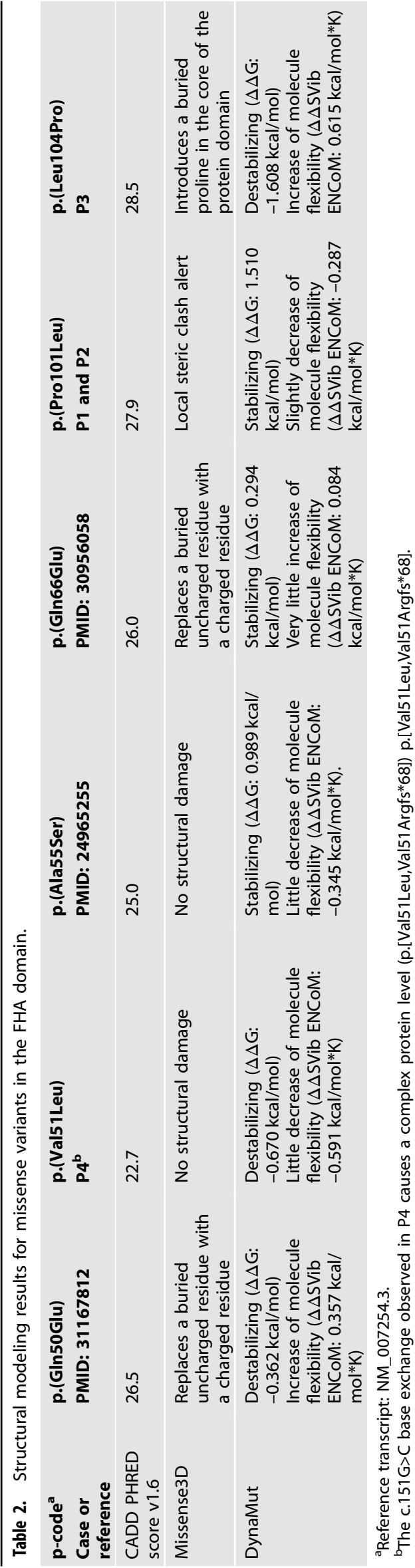

published aberrant splicing effect [24] of the maternally inherited canonical donor variant c. $1029+2 \mathrm{~T}>\mathrm{C}$, we performed RNA sequencing from PAXgene blood sample of P4 and both parents (Fig. 3). RNA sequencing data in the sample of P4 and the heterozygous carrier father (F3-f) showed mainly normal splicing events for the exon $2 / 3$ region with nucleotide exchange c.151G $>C$ leading to the missense change p.Val51Leu one consequence from this allele. The sequencing reads also support an aberrant transcript with retention of intron 2 (r.151_152ins151 +1 152-1), which is predicted to cause a frameshift and a premature stop codon (p.Val51Argfs*68). Overall the c.151G>C base exchange causes a complex effect on RNA and protein level (p.[Val51Leu,Val51 Argfs*68]) (Fig. 3B). Estimating the predominant aberrant transcript and effect is complicated by the presence of the second variant in the index and apparently incomplete nonsense mediated RNA-decay. Based on the allele fraction of the RNA-seq reads with the c.151G $>C$ change in the father $(10 / 35$ overall), the two consequences from this allele (4 correctly spliced with base exchange, 6 with retention) are about equally expressed.

For the variant c.1029+2T>C inherited from the mother (F3-m) our RNA-seq data confirmed the previously described [24] and expected effect of exon 11 skipping (r.937_1029del) leading to an inframe deletion of the 31 AAs (p.Phe313_Pro343del). We identified evidence for an intron 10 retention (r.936_937ins936+1_937-1) as a second aberrant transcript, which is predicted to cause a truncated protein (p.v) (Fig. 3C). Thus, also this canonical splice variant causes a complex mixture of aberrant transcripts (p.[Phe313_Pro343del, Leu312_Phe313ins*18]). Estimating the predominant effect is again complicated by multiple novel transcripts from both alleles in the index. From the spliced reads observed over the skipped exon in the mother (compare Fig. 3C), one would calculate an exon-inclusion ratio (or percent spliced in) of $56.8 \%(((30+33) / 2) /((30+33) / 2+$ 24)) for exon 11. To restore a model with equal expression of the transcripts from both alleles in the mother and assuming no nonsense mediated decay, 7.5 transcripts would have to be assigned to the retention event (add 7.5 to the denominator) which would constitute $\sim 11.9 \%(7.5 / 63)$ of all transcripts. This calculation is in agreement with the observed coverage profile between exon 10 and intron $10(11 / 69, \sim 15.9 \%)$. Overall, this confirms that the predominant aberrant splice effect caused by the c.1029+2T>C variant is in-frame exon skipping.

\section{DISCUSSION}

Before our report, PNKP was already associated with a wide phenotypic spectrum, which could be related to its multidomain architecture. The FHA domain recruits PNKP to DNA damage sites [25], where it is involved in repair of both single- and doublestrand breaks through its enzymatically active kinase and phosphatase domains $[26,27]$.

Despite the various PNKP-associated phenotypes [5-9, 28-30], prenatal presentations in humans, especially noticeable brain anomalies, were unreported to date. Our compilation of prenatal diagnostic procedures and fetal pathological examination of P1 and P2 revealed neurodevelopmental and neurodegenerative brain alterations comparable to those described in mouse models with neuronal tissue-specific inactivation of PNKP [31]. These include general hypoplasia of different cerebral and cerebellar regions, without a histologically recognizable neuronal migration disorder. Furthermore, the two fetuses showed a convincing phenotypic accordance with the previously described MCSZ phenotype [6]. While the exact pathomechanism of PNKPassociated microcephaly is still disputed, the prenatal manifestation represents the most severe outcome. This may arise from extreme genome instability in neurons with impaired development on the one hand and increased cell death on the other hand (e.g., extensive white matter deficit in P1). 
A

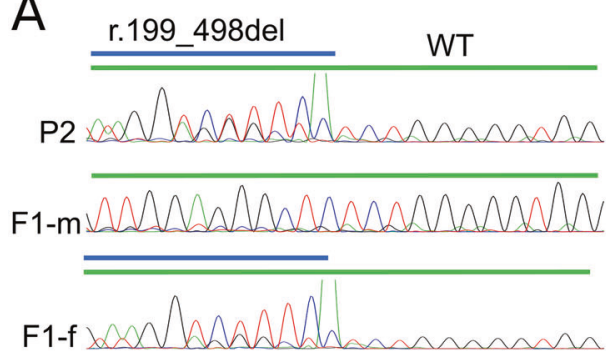

ᄁᄁ

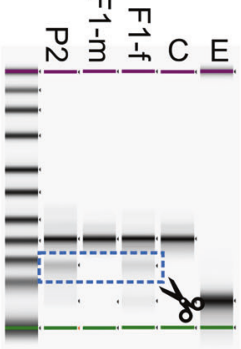

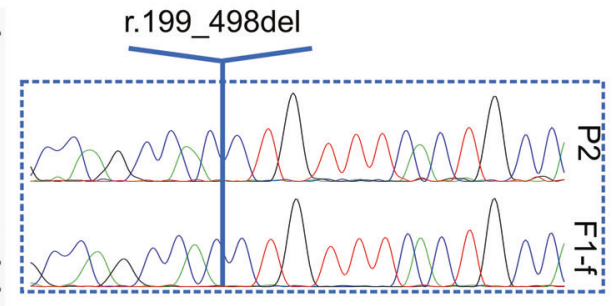

B

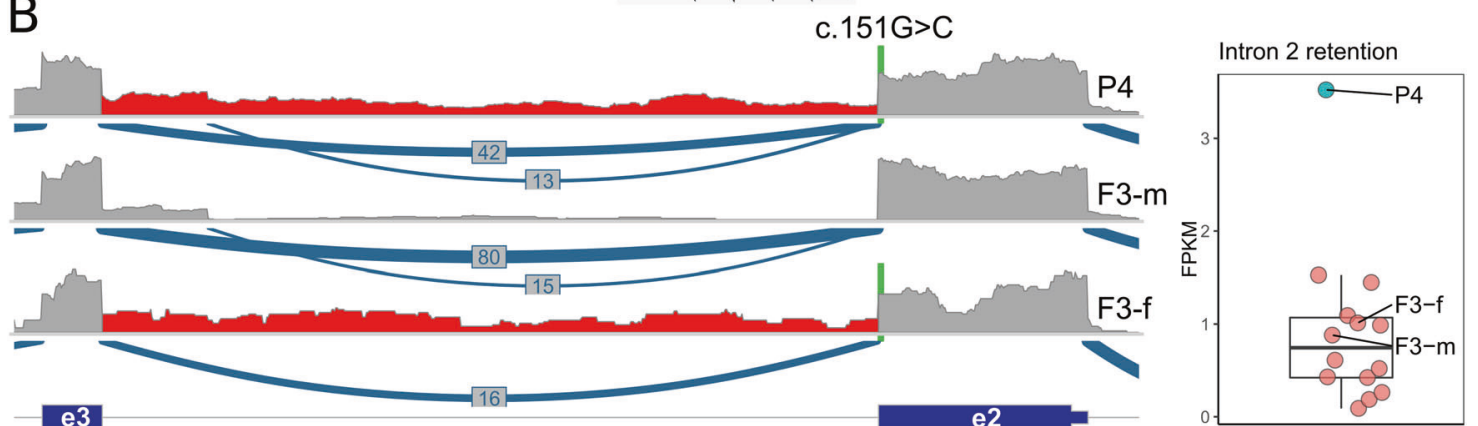

C

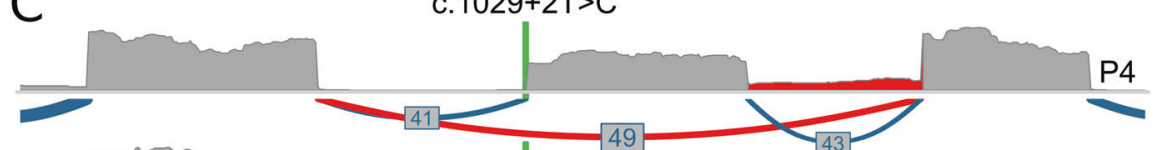

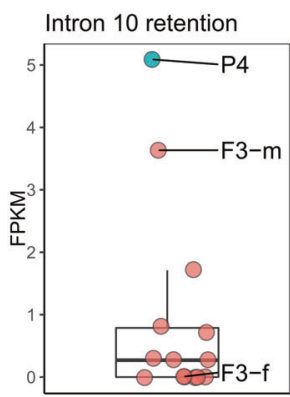

Fig. 3 RNA analyses. A RT-PCR and subsequent Sanger sequencing (left panel) on CDNA derived from RNA of individual P2 and both parents (F1-m, F1-f) to determine the effect of the c.498G>A variant. In P2 and the heterozygous carrier father a smaller product is present (middle panel). Right panel: skipping of exon 4 (r.199_498del) causes an in-frame deletion on protein level (p.Leu67_Lys166del). WT wildtype, C control, E empty. B Coverage and Sashimi-plot (left panel) of RNA-seq from family 3 for the exon 2-exon 3 region. The c.151G>C variant is indicated with a green line. Coverage plot for P4 and the heterozygous carrier father (F3-f) (highlighted in red), indicating intron 2 retention (r.151_152ins151+1_152-1). This is confirmed by the FPKM values shown as box and scatter plots (right side), where P4 is the only outlier. C Coverage and Sashimi-plot (left panel) of RNA-seq data from family 3 for the exon 10-exon 12 region. The position of the c.1029+2T>C variant is indicated with a green line. The Sashimi-plot shows skipping of exon 11 (r.937_1029del; highlighted in red) in P4 and the heterozygous carrier mother (F3-m), which is predicted to cause an in-frame deletion (p.Phe313_Pro343del). In addition, the coverage plot indicates retention of intron 10 (r.936_937ins936+1_937-1; red highlight) in P4 and F3-m, which is confirmed by the FPKM values for this exon (right panel and predicted to cause a truncated protein (p.Leu312_Phe313ins*18).

With suspicion of recurrent microcephaly, fetal autopsy of P2 was oriented toward an underlying syndromic disorder. Missing macroscopic signs for holoprosencephaly such as hypotelorism, hypoplastic anterior cranial fossae, or absent cribriform plate of ethmoid bone in the first pathological examination (limited on microscopic level due to autolysis) together with the unremarkable holoprosencephaly panel analysis provide sufficient arguments to retrospectively rule out holoprosencephaly in $\mathrm{P} 1$. The synergy of unbiased exome sequencing for prenatal anomalies and exact phenotyping in a syndrome-oriented fetal autopsy in P2 highlight their importance for diagnosing an unusual manifestation of a known disease and evaluation of a novel variant of uncertain clinical significance.

Given the OFC values observed immediately after birth in P3 and $\mathrm{P} 4$, the presence of prenatal microcephaly in these individuals is obvious. The diagnosis of PM relies on US measurements in comparison to distributions for the respective GA and exclusion of exogenous causes such as infectious diseases. Until the recent Zika virus outbreak, no international standards and guidelines had been defined and research on the diagnostic performance of US measurements for fetal microcephaly was hampered by the overall rarity of the condition [32]. Performance for prenatal US diagnosis seems good at the more extreme ends $(<-4$ SD) or when additional brain anomalies are present [32], like in the case of the two fetuses of family 1 . In addition, improved US technology and specialist training during the last years might have enabled these prenatal diagnoses.

We recommend interpreting the phenotypic presentations associated with biallelic variants in PNKP as continuous spectrum instead of the separated clinical entities MCSZ, AOA4, and CMT2B2, in agreement with previous suggestions [33]. In this disease model, our report adds the most severe, prenatal presentation with extreme microcephaly, and possibly unviable additional cerebral anomalies in the two sibling fetuses. With regard to the grade of severity, this is followed by postnatal microcephaly with severe to mild intellectual disability combined with further neurological symptoms like epilepsy and finally by adult-onset polyneuropathy as the mildest presentation (Fig. 4). 


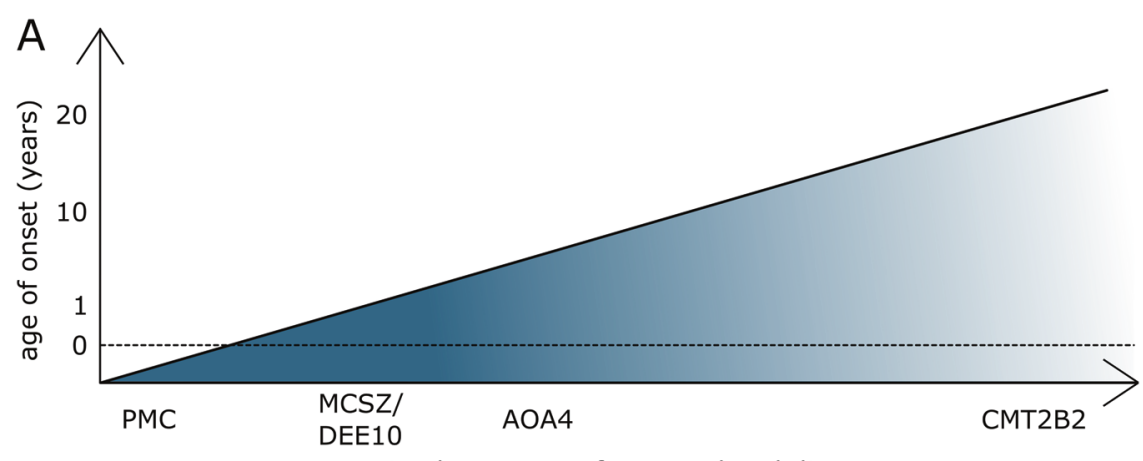

main phenotypes of $P N K P$-related disease

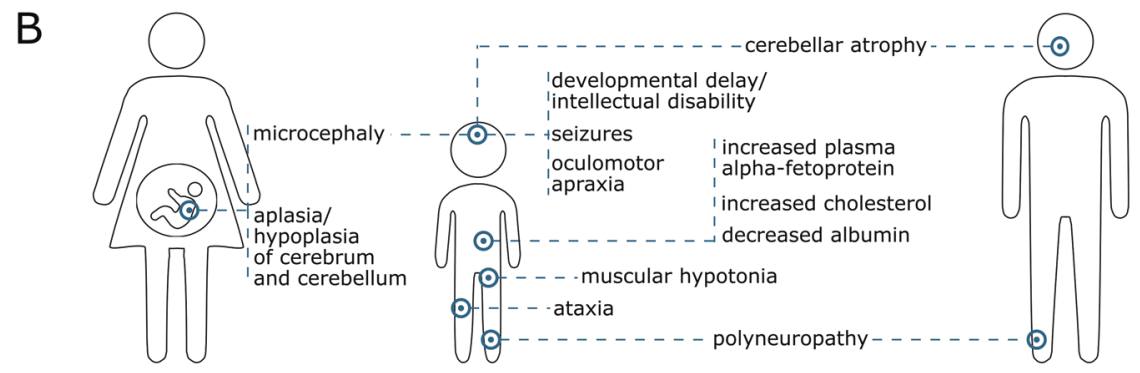

Fig. 4 Phenotypes. A Schematic indicating the proposed phenotypic continuum in PNKP-related disease: MCSZ (microcephaly, seizure, and developmental delay; MIM\# 613402) and developmental and epileptic encephalopathy (DEE10; MIM\# 613402), AOA4 (ataxia-oculomotor apraxia type 4; MIM\# 616267), and Charcot-Marie-Tooth disease, type 2B2 (CMT2B2; MIM\# 605589). Prenatal manifestation is added as prenatal microcephaly (PMC). Interpreted severity of the disease is shown by color course. B Comparison of the main clinical aspects between prenatal period (left), childhood (middle), and adulthood (right). Aplasia/hypoplasia of the cerebrum (HP:0007364) and the cerebellum (HP:0007360) is exclusively described in the prenatal period. Microcephaly (HP:0000252) is one of the main features of the prenatal and childhood phenotype MCSZ. MCSZ and DEE10 also comprise developmental delay/intellectual disability (HP:0001263; HP:0001249), seizures (HP:0001250), and muscular hypotonia (HP:0001252). Ataxia (HP:0001251) and oculomotor apraxia (HP:0000657) are characteristic for AOA4. Distinct biochemical features (elevated alpha-fetoprotein HP:0006254, hypoalbuminemia HP:0003073, hypercholesterolemia HP:0003124) were mostly described in cases with AOA4. Overlap between childhood and adulthood presentation are cerebellar atrophy (HP:0001272) and polyneuropathy (HP:0000763).

Observing this clinical variability, the question arises whether there could be a relation to genetic variant context.

On a genomic level, PNKP stands out given the specific composition with many small introns toward the $3^{\prime}$ end when compared to the average human intron size [34]. Smaller introns are associated with a higher likelihood of intron retention [35], a mechanism we proved for two novel variants here. Except the RTPCR analysis of the C.1029+2T>C variant, detailed RNA analyses of disease-associated PNKP variants were previously not reported, despite many intronic and splice sites affecting changes described $[6,24,25,28,33]$. The results of our RNA splicing analyses for one novel silent variant, one known splice donor variant and moreover one variant annotated as missense point toward a likely underappreciated pathomechanism. Predictions by computational tools implicate a splice effect for 15 of 43 (35\%) PNKP variants, which implies a need for further functional evaluation. We show that complex splice events in PNKP can readily be assessed using RNA from peripheral blood. Expanding these analyses to newly identified variants will support variant pathogenicity interpretation and improve understanding of aberrant splicing for genes with similar exon configuration.

Next to the often complex and possibly hidden splice effects identified here, true AA substitutions are to date the most difficult variants to interpret. Evaluation of missense variants can be composed of evolutionary conservation, functional predictions, and genetic context as it is used comprehensively, e.g., in the CADD score [36]. Here we complemented the analysis of such scores in the linear protein model with structural protein modeling. Four variants identified in the three families affect the FHA domain. The AA residues affected by the missense variants p.(Gln50Glu), p. [Val51Leu,Val51Argfs*68], p.(Ala55Ser), p.(Gln66Glu), p.(Pro101Leu), and p.(Leu104Pro) show significant clustering with other AA changes described in the literature. Interestingly, the compound heterozygous variants identified in family 1 alter the same region of PNKP: the variant p.Leu67_Lys166del predominantly results in a deletion affecting parts of the FHA, linker, and phosphatase domain and thereby encompasses a total of around $20 \%$ of PNKP, while the second is the missense variant p.(Pro101Leu) located at the C-terminus of the FHA domain. Previously, no disease-associated variants were described in this particular PNKP region, which shows high conservation and absence of homozygous variants in population databases. The severity of the observed prenatal phenotype may either due to the combined interdomain effect of the large in frame deletion or serves as an intriguing confirmation of a "Wald's domain" at the crossing between the FHA and linker domains, which has recently been proposed as a survivorship bias for disease-associated PNKP variants [11].

Taking into account the different mutational mechanisms of PNKP variants and the wide phenotypic spectrum, currently no clear genotype-phenotype correlation and therefore no accurate phenotypic prediction in association with a specific variant seems possible. While PNKP may be an extreme example, this is a common challenge in disorders with autosomal-recessive inheritance. In homozygous state, either because of consanguinity or due to founder variants, other effects of the haplotype can influence phenotype, while in compound heterozygous state the combination of two variants with possibly different effects each complicates accurate phenotype association. The latter is exemplified by the difference in clinical severity in individuals $\mathrm{P} 3$ and $\mathrm{P} 4$ who both carry the same splice variant c. $1029+2 T>C$ causing a mixture of protein effects (p.[Phe313_Pro343del, Leu312_Phe313ins*18]) on one allele and a different missense variant each on the second allele. Individual 
P4, who has the milder phenotype without seizures, carriers the splice region variant c.151G $>C$ causing mixture of missense ( $p$. Val51Leu) and truncating effect (p.Val51Argfs*68). In contrast, individual P3 who has severe neurodevelopmental delay, seizures, and extreme microcephaly $(<-6 S D)$ carries the missense variant c.311T >C, p.(Leu104Pro), which lies in the same FHA region and close proximity of the variants in family 1 . While this anecdotal correlation seems convincing, dysfunction of the phosphatase domain was already associated with PNKP-associated neurodevelopmental phenotype $[11,25]$, which we can support with variants in all three families affecting this domain. Systematic functional studies matched with standardized clinical assessment (HPO; see Fig. 4) will be needed to reach precise genotype-phenotype prediction.

Here, we extended the "PNKP-associated disorder continuum" to the prenatal period, complemented missense variant interpretation with 3D structure analysis and presented the first RNAseq data used to elucidate exact effects of PNKP variants. The knowledge of distinct fetal phenotypes will be helpful for genetic variant assessment, especially those with unknown significance. Only with knowledge of variant pathogenicity and expected symptoms, we will be able to improve counseling in the prenatal setting, management in the postnatal period, prenatal diagnosis in subsequent pregnancies, and finally enable potential future treatments.

\section{WEB RESOURCES}

gnomAD browser: http://gnomad.broadinstitute.org/,

Mutalyzer: https://mutalyzer.nl,

ClinVar: https://www.ncbi.nlm.nih.gov/clinvar/,

Missense3D: http://missense3d.bc.ic.ac.uk/missense3d/,

Dynamut: http://biosig.unimelb.edu.au/dynamut/,

Mutation3D: http://mutation3d.org/.

\section{DATA AVAILABILITY}

All data generated or analyzed during this study can be found either in the online version of this article at the publisher's website or has been uploaded to Zenodo.

\section{REFERENCES}

1. Opitz JM, Holt MC. Microcephaly: general considerations and aids to nosology. J Craniofac Genet Dev Biol. 1990;10:175-204.

2. Passemard S, Kaindl AM, Verloes A. Microcephaly. Handb Clin Neurol. 2013;111:129-41.

3. Peter JM. Maintaining genome stability in the nervous system. Nat Neurosci. 2013;16:1523-9.

4. Chappell C. Involvement of human polynucleotide kinase in double-strand break repair by non-homologous end joining. EMBO J. 2002;21:2827-32.

5. Karimi-Busheri F, Rasouli-Nia A, Allalunis-Turner J, Weinfeld M. Human polynucleotide kinase participates in repair of DNA double-strand breaks by nonhomologous end joining but not homologous recombination. Cancer Res. 2007:67:6619-25.

6. Shen J, Gilmore EC, Marshall CA, Haddadin M, Reynolds JJ, Eyaid W, et al. Mutations in PNKP cause microcephaly, seizures and defects in DNA repair. Nat Genet. 2010;42:245-9.

7. Bras J, Alonso I, Barbot C, Costa MM, Darwent L, Orme T, et al. Mutations in PNKP cause recessive ataxia with oculomotor apraxia type 4. Am J Hum Genet. 2015;96:474-9.

8. Carvill GL, Heavin SB, Yendle SC, McMahon JM, O'Roak BJ, Cook J, et al. Targeted resequencing in epileptic encephalopathies identifies de novo mutations in CHD2 and SYNGAP1. Nat Genet. 2013;45:825-30.

9. Leal A, Bogantes-Ledezma $S$, Ekici $A B$, Uebe $S$, Thiel $C T$, Sticht $H$, et al. The polynucleotide kinase $3^{\prime}$-phosphatase gene (PNKP) is involved in Charcot-MarieTooth disease (CMT2B2) previously related to MED25. Neurogenetics. 2018;19:215-25.

10. Pedroso JL, Rocha CRR, Macedo-Souza LI, De Mario V, Marques W, Barsottini OGP, et al. Mutation in PNKP presenting initially as axonal Charcot-Marie-Tooth disease. Neurol Genet. 2015;1:e30.
11. Bermúdez-Guzmán L, Jimenez-Huezo G, Arguedas A, Leal A. Mutational survivorship bias: the case of PNKP. PLoS One. 2020;15:e0237682.

12. Popp, Neuser. Data files for manuscript Prenatal phenotype of PNKP-related primary microcephaly associated with variants in the FHA and phosphatase domain. Zenodo. 2021. https://zenodo.org/record/5264692. Accessed 21 Jul 2021.

13. Hebebrand $M$, Hüffmeier $U$, Trollmann $R$, Hehr $U$, Uebe $S$, Ekici $A B$, et al. The mutational and phenotypic spectrum of TUBA1A-associated tubulinopathy. Orphanet J Rare Dis. 2019;14:38.

14. Richards S, Aziz N, Bale S, Bick D, Das S, Gastier-Foster J, et al. Standards and guidelines for the interpretation of sequence variants: a joint consensus recommendation of the American College of Medical Genetics and Genomics and the Association for Molecular Pathology. Genet Med. 2015;17:405-23.

15. Köhler S, Carmody L, Vasilevsky N, Jacobsen JOB, Danis D, Gourdine J-P, et al. Expansion of the Human Phenotype Ontology (HPO) knowledge base and resources. Nucleic Acids Res. 2019;47:D1018-27.

16. Potter EL, Craig JM. Pathology of the fetus and the infant. 3rd ed. Chicago: Year Book Medical Publishers; 1975. p. 15-24.

17. World Health Organization. WHO child growth standards: methods and development. Head circumference-for-age, arm circumference-for-age, triceps skinfold-for-age and subscapular skinfold-for-age. 2007.

18. Schoner K, Axt-Fliedner R, Bald R, Fritz B, Kohlhase J, Kohl T, et al. Fetal pathology of neural tube defects - an overview of 68 cases. Geburtshilfe Frauenheilkd. 2017;77:495-507.

19. Neuser S, Brechmann B, Heimer G, Brösse I, Schubert S, O'Grady L, et al. Clinical, neuroimaging, and molecular spectrum of TECPR2-associated hereditary sensory and autonomic neuropathy with intellectual disability. Hum Mutat. 2021;42:762-76.

20. Popp B, Erber R, Kraus C, Vasileiou G, Hoyer J, Burghaus S, et al. Targeted sequencing of $\mathrm{FH}$-deficient uterine leiomyomas reveals biallelic inactivating somatic fumarase variants and allows characterization of missense variants. Mod Pathol. 2020:33:2341-53.

21. Li H-D, Funk CC, Price ND. iREAD: a tool for intron retention detection from RNA seq data. BMC Genomics. 2020;21:128.

22. Ali AAE, Jukes RM, Pearl LH, Oliver AW. Specific recognition of a multiply phosphorylated motif in the DNA repair scaffold XRCC1 by the FHA domain of human PNK. Nucleic Acids Res. 2009;37:1701-12.

23. Snijders RJ, Nicolaides KH. Fetal biometry at 14-40 weeks' gestation. Ultrasound Obstet Gynecol. 1994;4:34-48.

24. Wai HA, Lord J, Lyon M, Gunning A, Kelly H, Cibin P, et al. Blood RNA analysis can increase clinical diagnostic rate and resolve variants of uncertain significance. Genet Med. 2020;22:1005-14.

25. Kalasova I, Hanzlikova H, Gupta N, Li Y, Altmüller J, Reynolds JJ, et al. Novel PNKP mutations causing defective DNA strand break repair and PARP1 hyperactivity in MCSZ. Neurol Genet. 2019;5:e320.

26. Tsukada K, Matsumoto $Y$, Shimada M. Linker region is required for efficient nuclear localization of polynucleotide kinase phosphatase. Sobol RW, editor. PLoS One. 2020;15:e0239404.

27. Bermúdez-Guzmán L, Leal A. DNA repair deficiency in neuropathogenesis: when all roads lead to mitochondria. Transl Neurodegener. 2019;8:14.

28. Caputi C, Tolve M, Galosi S, Inghilleri M, Carducci C, Angeloni A, et al. PNKP deficiency mimicking a benign hereditary chorea: The misleading presentation of a neurodegenerative disorder. Parkinsonism Relat Disord. 2019;64:342-5.

29. Poulton C, Oegema R, Heijsman D, Hoogeboom J, Schot R, Stroink H, et al Progressive cerebellar atrophy and polyneuropathy: expanding the spectrum of PNKP mutations. Neurogenetics 2013;14:43-51.

30. Dumitrache LC, McKinnon PJ. Polynucleotide kinase-phosphatase (PNKP) mutations and neurologic disease. Mech Ageing Dev. 2017;161:121-9.

31. Shimada M, Dumitrache LC, Russell HR, McKinnon PJ. Polynucleotide kinase-phosphatase enables neurogenesis via multiple DNA repair pathways to maintain genome stability. EMBO J. 2015;34:2465-80.

32. Chibueze EC, Parsons AJQ, Lopes K, da S, Yo T, Swa T, et al. Diagnostic accuracy of ultrasound scanning for prenatal microcephaly in the context of Zika Virus infection: a systematic review and meta-analysis. Sci Rep. 2017;7:2310.

33. Gatti M, Magri S, Nanetti L, Sarto E, Di Bella D, Salsano E, et al. From congenital microcephaly to adult onset cerebellar ataxia: distinct and overlapping phenotypes in patients with PNKP gene mutations. Am J Med Genet A. 2019;179:2277-83.

34. Abebrese EL, Ali SH, Arnold ZR, Andrews VM, Armstrong K, Burns L, et al. Identification of human short introns. PLoS One. 2017;12:e0175393.

35. Monteuuis G, Wong JJL, Bailey CG, Schmitz U, Rasko JEJ. The changing paradigm of intron retention: regulation, ramifications and recipes. Nucleic Acids Res. 2019;47:11497-513.

36. Rentzsch P, Witten D, Cooper GM, Shendure J, Kircher M. CADD: predicting the deleteriousness of variants throughout the human genome. Nucleic Acids Res. 2019;47:D886-94. 


\section{ACKNOWLEDGEMENTS}

We thank all involved families for participating in this study. We thank Viktoria Wischmann (medical laboratory assistant, Institute of Pathology, Philipps University of Marburg) for engaged collaboration and excellent technical support in performing RNA extraction for P2 as well as Mirko Wegscheider (Department of Neurology, University of Leipzig) for his kind beneficial assistance with interpretation of MRI images.

\section{AUTHOR CONTRIBUTIONS}

BP conceived the initial study concept. SN and IK coordinated collection of clinical and genetic data through matchmaking and personal communications. SN and IK reviewed literature data and standardized the clinical HPO terms. IK, AS, RAJ, TB, JD, $S$ Syrbe, MP, SR, CR, HR, and KS provided clinical and genetic data and performed clinical assessments. KS and HR performed fetal pathology analysis. KS provided fetal pathological images and fetal RNA samples. S Schubert conducted RT-PCR analyses. $\mathrm{DLD}, \mathrm{MR}$, and $\mathrm{BP}$ analyzed RNA-seq data. LB-G, SN, BP, and AL performed structural protein analysis. SN, AL, LB-G, and BP created main figures and the Supplementary materials. SN, IK, and BP wrote and edited the manuscript. All authors reviewed, commented, and agreed on the final draft manuscript.

\section{FUNDING}

BP is supported by the Deutsche Forschungsgemeinschaft (DFG) through grant PO2366/2-1. DLD is funded through "Clinician Scientist Programm, Medizinische Fakultät der Universität Leipzig." Open Access funding enabled and organized by Projekt DEAL.

\section{COMPETING INTERESTS}

The authors declare no competing interests.

\section{ETHICS APPROVAL AND CONSENT TO PARTICIPATE}

The Ethical Committee of the Medical Faculty, Leipzig University, approved genetic testing in a research setting for all probands (224/16-ek and 402/16-ek). Written consent of the parents to publish genetic and clinical data, postmortem images (P1, $\mathrm{P} 2)$, sonography (P1, P2), and magnetic resonance imaging (MRI) (P1, P3, and P4) was received and archived by the authors.

\section{ADDITIONAL INFORMATION}

Supplementary information The online version contains supplementary material available at https://doi.org/10.1038/s41431-021-00982-y.

Correspondence and requests for materials should be addressed to Sonja Neuser or Bernt Popp.

Reprints and permission information is available at http://www.nature.com/ reprints

Publisher's note Springer Nature remains neutral with regard to jurisdictional claims in published maps and institutional affiliations.
Open Access This article is licensed under a Creative Commons Attribution 4.0 International License, which permits use, sharing, adaptation, distribution and reproduction in any medium or format, as long as you give appropriate credit to the original author(s) and the source, provide a link to the Creative Commons license, and indicate if changes were made. The images or other third party material in this article are included in the article's Creative Commons license, unless indicated otherwise in a credit line to the material. If material is not included in the article's Creative Commons license and your intended use is not permitted by statutory regulation or exceeds the permitted use, you will need to obtain permission directly from the copyright holder. To view a copy of this license, visit http://creativecommons. org/licenses/by/4.0/.

(c) The Author(s) 2021 